Research article

Open Access

\title{
Investigation of the Volatile Fraction of Rosemary Infusion Extracts
}

\author{
Christine TschIggerL, Franz BUCAR *
}

Institute of Pharmaceutical Sciences, University of Graz, Universitätsplatz 4/1, 8010, Graz, Austria

* Corresponding author. E-mail: franz.bucar@uni-graz.at (F. Bucar)

Sci Pharm. 2010; 78: 483-492

doi:10.3797/scipharm.1004-23

Published: June $16^{\text {th }} 2010$

Accepted: $\quad$ June $16^{\text {th }} 2010$

Received: $\quad$ April $29^{\text {th }} 2010$

This article is available from: http://dx.doi.org/10.3797/scipharm.1004-23

(c) Tschiggerl and Bucar; licensee Österreichische Apotheker-Verlagsgesellschaft m. b. H., Vienna, Austria.

This is an Open Access article distributed under the terms of the Creative Commons Attribution License (http://creativecommons.org/licenses/by/3.0/), which permits unrestricted use, distribution, and reproduction in any medium, provided the original work is properly cited.

\begin{abstract}
The relative proportions of chemical classes (hydrocarbons, oxides, alcohols, ketones, esters) in the essential oil of rosemary (Rosmarinus officinalis L., Lamicaeae) and in the volatile fraction of the infusion extracts were examined and showed remarkable differences.

The volatile compounds of the infusion were isolated by two different methods, hydrodistillation and solid phase extraction (SPE). The main constituents of the volatile fraction of the infusion were (hydrodistillation/SPE): 1,8-cineole $(42.4 \% / 44.7 \%)$, camphor $(31.4 \% / 31.8 \%)$, a-terpineol $(8.6 \% / 8.1 \%)$ and borneol $(8.3 \% / 7.8 \%)$. The qualitative and quantitative composition of the volatile compounds of the infusion was compared to the essential oil isolated by hydrodistillation directly from the leaves. The major constituents of the essential oil of the leaves were 1,8-cineole $(41.6 \%)$, camphor $(17.0 \%)$, a-pinene $(9.9 \%)$, a-terpineol (4.9\%) and borneol (4.8\%). Comparison of the total essential oil yield quantified by hydrodistillation of the infusion $(0.36 \% \mathrm{v} / \mathrm{w})$ with the essential oil yield of the leaves $(1.84 \% \mathrm{v} / \mathrm{w})$ revealed that only $19.6 \%$ of the initial oil could be extracted by infusion.
\end{abstract}

\section{Keywords}

Rosmarinus officinalis • Infusion • GC-MS • Essential oil • SPE

\section{Introduction}

Many herbs with potentially beneficial effects which are attributed to the volatile constituents are used as herbal teas, for instance peppermint, lemon balm, thyme or rosemary. In traditional medicine, Rosmarinus officinalis L. (Lamiaceae) leaves are used 
as an integrant of various tea mixtures (up to $350 \mathrm{mg} / \mathrm{g}$ tea) in cardiovascular, sleep and neuritic disorders [1]. Rosemary has also gained much interest during the last years due to its role as antioxidant in food [2-4]. The most characteristic constituents of the leaves are essential oil, phenolic diterpenes, rosmarinic acid derivatives, flavonoids, triterpenes and steroids [1,5]. The essential oil composition was subject to various previous studies [6-8]. According to these data the following compounds are found as the main constituents in $R$. officinalis essential oil (REO), considering the fact that compounds vary in chemotype and origin: 1,8-cineole, camphor, $\alpha$-pinene, camphene, borneol, bornyl acetate, myrcene, limonene, a-terpineol and caryophyllene [1, 5, 9]. Antimicrobial activity, antitumor activity, antispasmodic and anticonvulsant activities and hyperglycemic effects were recognized as pharmacological actions of REO [10,11].

Antibacterial effects of REO have been reported against different gram positive and gram negative strains [12]. The antimicrobial effects of REO against Staphylococcus aureus, Pseudomonas aeroginosa and Candida albicans were mainly attributed to 1,8-cineole, whereas the effect against Escherichia coli was related to monoterpene hydrocarbons [13]. Apart from this, REO shows antioxidative activity which seems to be higher or comparable to that of a-tocopherol [12]. It was reported that these effects are the synergistic result of the oil composition [14]. The antioxidant activity of REO has been compared with that of Thymus vulgaris and has been shown to be almost as efficient [15].

$R$. officinalis leaves infusion is widely used in folk medicine as well as for food purposes, however its aromatic composition is only insufficiently investigated [16], a fact which is also true for many other herbal teas.

Therefore, the aim of this study was to compare the essential oil from rosemary infusion extract isolated by two different methods (hydrodistillation versus SPE) and to determine the qualitative and quantitative composition of the volatile compounds from infusion versus original rosemary essential oil (REO).

\section{Results and Discussion}

The composition of the essential oil of $R$. officinalis and that of the volatile fraction of the rosemary infusion obtained by hydrodistillation as well as by SPE were compared.

The qualitative and quantitative results of REO which were in conformity with data from literature are indicated in Table 1 [6-8]. The essential oil yield was $1.84 \% \pm 0.036$ (v/w) and its composition was established by $97.4 \%$ of its total peak area from the GC-MS chromatograms. Thirty-eight compounds were identified. The main constituents of the essential oil were 1,8-cineole, camphor, a-pinene, a-terpineol and borneol. The other constituents were present in levels of less than $4 \%$.

The aromatic composition of the infusion extracts were established by $98.4 \%$ (hydrodistillation) and by $98.9 \%$ (SPE) of total peak area from the GC-MS chromatograms. More than $90 \%$ of the volatile fraction of the infusion extracts was formed by its main constituents 1,8-cineole, camphor, a-terpineol and borneol. All other constituents were present in levels of less than $3 \%$. Detailed data and standard deviations are shown in Table 1. 
Tab. 1. Percentage composition ${ }^{a}$ of the essential oil of Rosmarinus officinalis and of the volatile fraction of the infusion extracts

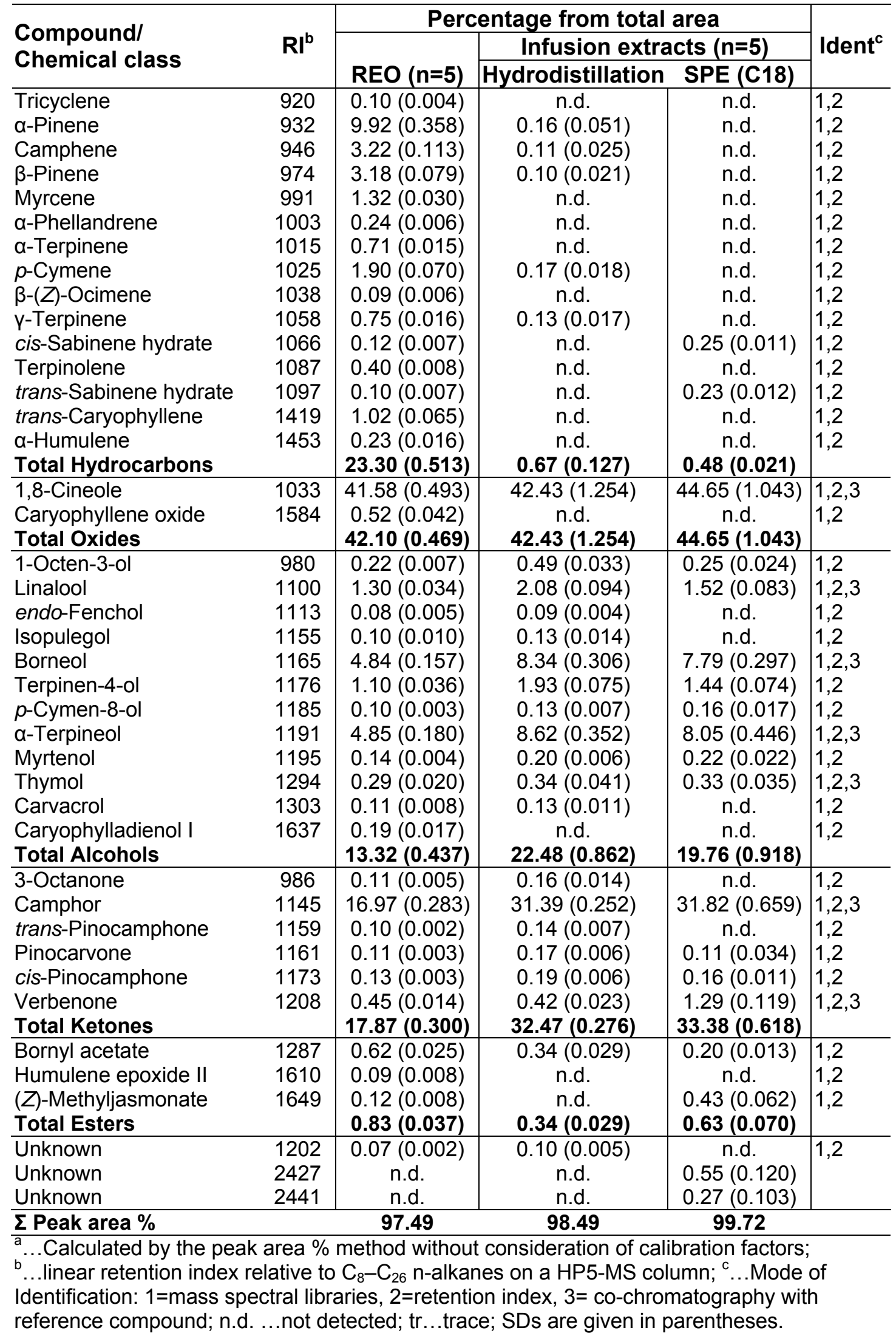


Furthermore, the percentages of hydrocarbons, oxides, alcohols, ketones and esters were calculated (Table 1). Oxides were mainly represented by 1,8 -cineole, more than $70 \%$ of the oil consisted of oxygen containing compounds.

When we compared the chromatograms of the infusion extracts obtained by hydrodistillation and SPE using qualitative and semiquantitative methods, we found no major differences. However, we noticed significant differences when we focused on the relative proportions of chemical classes of the constituents determined in REO in comparison with both infusion extracts.

In the infusion extracts the amount of hydrocarbons decreased whereas the total level of alcohols and ketones increased. The amount of esters appeared to be inconsiderable in REO and in the infusion extracts. The ratio of oxides in relation to other compound classes remained on a comparable level in both, infusion and REO. The proportion of oxygen containing compounds increased from $74.12 \%$ (REO) to $97.72 \%$ (infusion, hydrodistillation) and $98.42 \%$ (infusion, SPE), respectively, based on \% of total peak area.

A plausible explanation by the means of octanol-water partition coefficient and of the boiling points of the major compounds could be attempted.

The significant loss of monoterpene hydrocarbons present in REO can be explained by their higher volatility and lower water solubility (boiling points at about $160^{\circ} \mathrm{C}$ and $\log P$ values of about 4.4-4.6 for a-pinene, camphene, $\beta$-pinene, myrcene, $p$-cymene, Y-terpinene [17]. The loss of the sesquiterpene hydrocarbon trans-caryophyllene (boiling point at $262^{\circ} \mathrm{C}$ and $\log \mathrm{P}$ value of 6.777 , at $25^{\circ} \mathrm{C}$ [17]), present in REO, can only be explained by its lipophility. The higher hydrophility and the less volatility of alcohols and ketones might also explain the fact that although the proportion of oxygen containing compounds significantly increased in the infusion extracts, the ratio oxides vs. alcohol + ketones shifted from 1.35 (REO) to 0.77 (infusion, hydrodistillation) and 0.84 (infusion, SPE), respectively (mean bp of alcohols and ketones $>205^{\circ} \mathrm{C}$ ). According to log $\mathrm{P}$ values, hydrophility of 1,8-cineole, $\alpha$-terpineol and borneol (the latter two represent the major alcoholic compounds in REO) are comparable (2.821, 2.790 and 2.707, respectively, at $25^{\circ} \mathrm{C}$ [17]), camphor, the main ketone also shows a similar log $P$ value of 2.128 (at $25^{\circ} \mathrm{C}$ ) [17]. A study concerning lemon verbena tea showed an increase of aldehyds and esters and decreasing amounts of hydrocarbons, oxides and alcohols [18].

The enrichment of camphor compared to 1,8-cineole (4:3 in infusions, 5:2 in REO) was even more pronounced in a study where the ratio of the two major compounds, 1,8-cineole and camphor, of 9:1 (infusion) and 5:2 (REO) was found, respectively [16]. Certainly, the relations vary according to chemotype and origin of the plant material. The two compounds 1,8-cineole and camphor amounted up to $70 \%$ of the whole essential oil present in the infusion as it also was noticed in the same study, where dissolution rate and kinetics of these two main compounds were studied precisely, but all the other constituents were ignored.

However, some minor compounds which were found in the essential oil obtained by hydrodistillation from the infusion were not detected in the chromatogram of the SPE extract and vice versa, see Table 1 . It is obvious that through infusion these highly volatile compounds were lost. Many of the monoterpene hydrocarbons as tricyclene, myrcene, 
a-phellandrene, a-terpinene, $p$-cymene, ocimene, terpinolene and the sesquiterpene hydrocarbons trans-caryophyllene and $\alpha$-humulene occurring in REO were not detected in the infusion. This loss can be explained by the tea preparation procedure with boiling water and was also observed in a study concerning Salvia officinalis infusion [19].

Recovery rates and results of the quantitative analysis of the volatile fraction obtained by SPE procedure are shown in Table 2. Mean values of the individual compounds and SDs $(n=5)$ were calculated. Calibration data of the compounds (Table 2) indicated linearity of the detector signal within the concentration range injected $\left(R^{2} \geq 0.9956\right)$.

Tab. 2. Quantification of the major volatile compounds of rosemary leaves infusion and calibration data*

\begin{tabular}{|c|c|c|c|c|}
\hline $\begin{array}{c}\text { Compound } \\
\text { name }\end{array}$ & Regression equation & $\mathbf{R}^{\mathbf{2}}$ & $\begin{array}{c}\text { Recovery rate\% } \\
(n=3)\end{array}$ & $\begin{array}{c}\mathrm{mg} / 100 \mathrm{ml} \text { infusion } \\
(\mathrm{n}=5)\end{array}$ \\
\hline 1,8-Cineole & $y=0.6347 x+0.1294$ & 0.9967 & $75.2(6.14)$ & $1.772(0.2391)$ \\
\hline Camphor & $y=0.5888 x+0.0953$ & 0.9958 & $70.9(7.00)$ & $1.241(0.1518)$ \\
\hline$\alpha$-Terpineol & $y=0.6922 x+0.0926$ & 0.9978 & $60.8(3.03)$ & $0.430(0.0485)$ \\
\hline Borneol & $y=0.7787 x+0.0397$ & 0.9956 & $81.3(3.32)$ & $0.350(0.0351)$ \\
\hline Terpinen-4-ol & $y=0.6922 x+0.0926$ & 0.9978 & $60.8(3.03)$ & $0.077(0.0082)$ \\
\hline Linalool & $y=0.6899 x+0.1106$ & 0.9975 & $70.6(3.97)$ & $0.069(0.0062)$ \\
\hline Verbenone & $y=0.9101 x+0.0999$ & 0.9989 & $88.4(5.06)$ & $0.062(0.0056)$ \\
\hline$\Sigma$ & & & & $4.001(0.4816)$ \\
\hline
\end{tabular}

Comparison of the total essential oil yield quantified by hydrodistillation of the infusion $0.36 \% \pm 0.040(\mathrm{v} / \mathrm{w})$, with the essential oil content of the leaves $1.84 \% \pm 0.036(\mathrm{v} / \mathrm{w})$, obtained by Clevenger apparatus, revealed that only $19.6 \%$ of the initial oil could be extracted by hydrodistillation of the infusion. Similarly, a low portion of volatiles was noticed in a study where Roman chamomile tea was investigated [20]. The original infusion after 3 hours hydrodistillation was also verified if there were traces of volatiles by doing an SPE extraction and GC-MS analysis. We found only traces of the major compounds around the detection limit. A total essential oil content of $4.26-4.38 \mathrm{mg} / 100 \mathrm{ml}$ infusion could be calculated based on an average relative density of $0.895-0.920$ for REO [21]. Compared to the recommended daily dose of 10-20 drops of essential oil, which corresponds to 190$380 \mathrm{mg}$, the recommended daily two or three cups of rosemary tea only contain a small proportion (6.39-6.57 mg per cup) of these active volatiles [1, 5, 22]. Similar amounts of essential oil, 1.3 to $11.4 \mathrm{mg}$ per cup of tea, where found in different fennel teas [23].

As a conclusion, SPE seems to be an elegant alternative to hydrodistillation and a comparable but more economic method for determining the aromatic composition of infusion extracts. The volatility of the individual compounds has a significant influence on their extractability during preparation of an infusion and significant losses of volatiles, above all hydrocarbons, can be expected. The fact that the volatile fraction of rosemary tea differs considerably compared to the original rosemary essential oil seems to be pharmaceutically relevant. 


\section{Experimental}

\section{Plant Material}

A commercial sample $R$. officinalis leaves was obtained from Mag. Kottas, Vienna (Austria). The material complied with the monograph of the European Pharmacopoeia [21]. A voucher specimen is kept at the Department of Pharmacognosy, Institute of Pharmaceutical Sciences, University of Graz.

\section{Chemicals}

All reagents and solvents used were of analytical or HPLC grade. Solvents and materials were purchased from the following suppliers: n-hexane (Fluka, Switzerland), methanol (Merck, Germany), methylene chloride (Roth, Germany), sodium sulfate (Roth, Germany). Authentic standards were 1,8-cineole (Fluka, Switzerland), (1R)-(+)-campher (Aldrich, Germany), linalyl acetate (Fluka, Switzerland), (+)-borneol (Fluka, Switzerland), (-)-verbenone (Fluka, Switzerland), (+)-a-terpineol (Fluka, Switzerland), (+/-)-linalool (Roth, Germany). The n-alkanes $\mathrm{C}_{8}-\mathrm{C}_{26}$ for the determination of the linear retention index were from Sigma, USA. Isolute C18 (EC) columns $(1 \mathrm{~g}, 15 \mathrm{ml})$ from Biotage, Sweden were used as SPE cartridges.

\section{Gas chromatography - mass spectrometry}

The composition of the essential oil and of the volatile compounds of the infusion extracts was determined by GC/MS. Each sample was analyzed ternary. Analyses were performed using an Agilent 7890A GC system coupled with an Agilent 5975C MSD operating at 70 $\mathrm{eV}$, ion source temperature $230^{\circ} \mathrm{C}$, interface temperature $280^{\circ} \mathrm{C}$. A split injection (split ratio, $80: 1$ ) at $240^{\circ} \mathrm{C}$ injector temperature was utilized. Injection volumes were $1 \mu \mathrm{l}$. A fused silica capillary column 5\% phenylmethylsiloxane (HP-5MS $30 \mathrm{~m} \times 250 \mu \mathrm{m} \times 0,25 \mu \mathrm{m}$, Agilent J \& W, USA) was used. The temperature program was as follows: 2 min at $45^{\circ} \mathrm{C}$, then to $250^{\circ} \mathrm{C}$ at $4^{\circ} \mathrm{C} / \mathrm{min}$, finally held at $250^{\circ} \mathrm{C}$ for $2 \mathrm{~min}$. The carrier gas was helium 5.6 at a flow rate $0.9 \mathrm{ml} / \mathrm{min}$. Data acquisition was performed with Agilent GC/MSD ChemStation Version E.02.00 for the mass scan range 40-300u.

Compounds were identified by retention indices [24] and by comparing their mass spectra with spectral data libraries [24, 25] and a laboratory own data base. Furthermore, for some compounds pure standard substances were available.

\section{Essential oil hydrodistillation}

Hydrodistillation procedures were done according to the European Pharmacopoeia [21]. $25 \mathrm{~g}$ of rosemary leaves were hydrodistilled for 3 hours. The infusion $(4500 \mathrm{ml})$ was also hydrodistilled for 3 hours immediately after preperation to avoid loss of volatiles. Five separate analyses were performed of each experiment. The essential oil samples were dried over anhydrous $\mathrm{Na}_{2} \mathrm{SO}_{4}$ and stored in dark glass bottles at $-20^{\circ} \mathrm{C}$ until analysis. The oil samples were diluted with hexane (1:30) before GC/MS analysis.

\section{Preparation of rosemary infusion}

An infusion was prepared according to literature [1]. Boiling distilled water $(4500 \mathrm{ml})$ was poured onto rosemary leaves $(60 \mathrm{~g})$, and the infusion was left to brew for $15 \mathrm{~min}$. Then it was filtered and rinsed three times with distilled water and brought to a final volume of 
exactly $4500 \mathrm{ml}$. These high amounts of infusion were necessary to determine the quantitative essential oil content with the Clevenger apparatus. The same tea preparation procedure with $450 \mathrm{ml}$ boiling water and $6 \mathrm{~g}$ leaves was done before SPE extraction. Each experiment was performed fivefold.

\section{Solid phase extraction (SPE) of the infusion}

The following method was adapted from literature [19]. An Isolute C18 (EC) solid phase extraction cartridge $(1 \mathrm{~g})$ was conditioned twice with $8 \mathrm{ml}$ methylene chloride and twice with $8 \mathrm{ml}$ methanol. Afterwards $8 \mathrm{ml}$ of distilled water was passed through the cartridge twice and not allowed to dry before the filtered infusion was applicated. The infusion was loaded onto the cartridge with a flow of 1-2 $\mathrm{ml} / \mathrm{min}$. The cartridge was dried for $15 \mathrm{~min}$ by putting on a slight vacuum. The compounds retained on the SPE column were eluted into a $5 \mathrm{ml}$ graduated flask with exactly $5 \mathrm{ml}$ of methylene chloride, which contained the internal standard, linalyl acetate $(200 \mathrm{ng} / \mu \mathrm{l})$. Pure dichloromethane was added to a final volume of $5 \mathrm{ml}$. Five separate determinations were performed. These samples were stored in glass bottles at $-20^{\circ} \mathrm{C}$ until they were used for GC/MS analysis.

\section{Semiquantitative analysis}

REO and the essential oil from the infusion obtained by hydrodistillation were quantified by the area\% method without considering calibration factors. For comparison, the SPE extract from the infusion was quantified with the same method, without considering the internal standard.

\section{Quantitative analysis}

Quantification and determination of recovery rates using the method of internal standard were done for the major compounds in the extract obtained by SPE. Standard solutions were prepared containing 50, 250, 500, 750 and $1000 \mathrm{ng} / \mu \mathrm{l}$ borneol, verbenone, camphor, a-terpineole, linalool and the same quantity $(200 \mathrm{ng} / \mu \mathrm{l})$ of the internal standard linalyl acetate, a substance which was absent in REO and also in the infusion extracts and which peak did not interfere with other substances in the chromatograms.

Four 1,8-cineole standard solutions were prepared containing 100, 300, 600, 900, 1200 $\mathrm{ng} / \mu \mathrm{l}$ and the same quantity of the internal standard $(200 \mathrm{ng} / \mu \mathrm{l})$. Within this range of concentrations the detector response was linear. The compounds for the standard solutions were dissolved in hexane. These standard solutions were used for creating calibration curves by linear regression (peak area compound / peak area internal standard versus concentration compound / concentration internal standard).

Quantity of each major constituent was calculated by using the following formula:

$$
\mathrm{m}_{\mathrm{i}}=\frac{\left(\text { amount }_{\text {lstd }}\right) \times\left(\text { area }_{i}\right) \times r f_{i}}{\text { area }_{\text {Istd }}}
$$

where $m_{i}$ is the total amount of substance $i$ in the sample (ng), amount $t_{\text {std }}$ the amount of internal standard which was added to the sample (ng), area $a_{i}$ the peak area of the substance $\mathrm{i}, r f_{i}$ the response factor obtained from the slope of the calibration curve and are $_{\text {Istd }}$ the peak area of the internal standard [26]. 
Determination of the recovery rates for the major compounds found in the SPE extract were done by adapting the SPE procedure according to section 2.6. Instead of the original infusion an essential oil free infusion, obtained by boiling for 2 hours to eliminate the volatiles, was used. Elution was done with $5 \mathrm{ml}$ of methylene chloride, which contained the internal standard linalyl acetate $(200 \mathrm{ng} / \mathrm{\mu l})$ and the same amount of borneol, 1,8-cineole, camphor, $\alpha$-terpineol, verbenone and linalool. Calculation was done by using the following formula :

$$
\mathrm{RR}=\frac{\left(\text { area }_{i}\right) \times\left(\text { amount }_{\text {Istd }}\right) \times 100 \times r f_{i}}{\left(\text { area }_{\text {Istd }}\right) \times\left(\text { amount }_{i}\right)}
$$

$R R$ is the recovery rate of substance $\mathrm{i}$ in percent, for other abbreviations see above.

\section{Authors' Statement}

\section{Competing Interests}

The authors declare no conflict of interest.

\section{References}

[1] Wichtl M, editor.

Rosmarini Folium

In: Teedrogen und Phytopharmaka. 5th ed.

Stuttgart: Wissenschaftliche Verlagsgesellschaft mbH, 2009: 573-575.

[2] Jamila B, Mohamed A, Abbas KA, Rahman RA, Karim R.

A review on the effect of animal diets and presence of selected natural antioxidants on lipid oxidation of meat.

J Food Agric Environ. 2009; 7: 76-81.

[3] Hasani-Ranjbar S, Larijani B, Abdollahi M.

A systematic review of the potential herbal sources of future drugs effective in oxidant-related diseases.

Inflamm Allergy Drug Targets. 2009; 8: 2-10.

doi:10.2174/187152809787582561

[4] Pokorny J.

Application of phenolic antioxidants in food products.

Electron J Environ Agric Food Chem. 2008; 7: 3320-3324.

[5] Blaschek W, Ebel S, Hackenthal E, Holzgrabe U, Keller K, Reichling J, Schulz V, editors.

Rosmarinus.

In: Hagers Enzyklopädie. 6th ed. Volume 13,

Stuttgart: Wissenschaftliche Verlagsgesellschaft mbH, 2007: 951-969.

[6] Kartnig T, Fischer U, Bucar F.

Vergleichende gaschromatographische Untersuchungen an ätherischen Wacholderölen, Fenchelölen und Rosmarinölen.

Sci Pharm. 1998; 66: 237-252.

[7] Kubeczka KH, Formacek V.

Essential oils analysis by capillary gas chromatography and carbon 13-NMR spectroscopy. 2nd ed. Chichester: John Wiley \& Sons, 2002: 285-300.

[8] Lawrence BM.

Progress in essential oils.

Perfum Flav. 2007; 32: 50-54. 
[9] Barnes J, Anderson LA, \& Phillipson JD.

Rosemary

In: Herbal Medicines. 3rd ed.

London: Pharmaceutical Press, 2007: 508-511.

[10] Bradley PR, editor

Rosemary leaf

In: British Herbal compendium: A Handbook of Scientific Information on widely used plant drugs.

Volume. 2.

Bournemouth: British Herbal Medicine Association, 2006: 333-338.

[11] Rosmarini folium.

In: ESCOP Monographs: The Scientific Foundation for Herbal Medicinal Products. 2nd ed.

Stuttgart: Georg Thieme Verlag, 2003: 429-436

[12] Baratta TM, Dorman DHJ, Deans SG, Figueiredo CA, Barroso JG, Ruberto G.

Antimicrobial and antioxidant properties of some commercial essential oils.

Flavour Frag J. 1998; 13: 235-244.

[13] Jirovetz L, Buchbauer G, Denkova Z, Stoyanova A, Murgov I, Schmidt E, Geissler M.

Antimicrobial testings and gas chromatographic analysis of pure oxygenated monoterpenes $1,8-$ cineole, $\alpha$-terpineol, terpinen-4-ol and camphor as well as target compounds in essential oils of pine (Pinus pinaster), rosemary (Rosmarinus officinalis), tea tree (Melaleuca alternifolia).

Sci Pharm. 2005; 73: 27-39.

[14] Wang W, Wu N, Zu YG, Fu YJ.

Antioxidative activity of Rosmarinus officinalis L. essential oil compared to its main components.

Food Chem. 2008; 108: 1019-1022.

doi:10.1016/j.foodchem.2007.11.046

[15] Sacchetti G, Maietti S, Muzzoli M, Scaglianti M, Manfredini S, Radice M, \& Bruni R.

Comparative evaluation of 11 essential oils of different origin as functional antioxidants, antiradicals

and antimicrobials in foods.

Food Chem. 2005; 91: 621-632.

doi:10.1016/j.foodchem.2004.06.031

[16] Schneider K, Pulver G, Kubelka W.

Infusions of Rosemary leaves: Dissolution rate and kinetics of the main volatile compounds.

Planta Med. 1992; 58: A678-A679.

[17] SciFinder (2009) American Chemical Society.

Data were calculated using Advanced Chemistry Development (ACD/Labs) Software V9.04 for Solaris. http://scifinder.cas.org/scifinder/

[18] Carnat A, Carnat AP, Fraisse D, Lamaison JL.

The aromatic and polyphenolic composition of lemon verbena tea.

Fitoterapia. 1999; 70: 44-49.

doi:10.1016/S0367-326X(98)00016-1

[19] Radulescu V, Chiliment S, Oprea E.

Capillary gas chromatography-mass spectrometry of volatile and semi-volatile compounds of Salvia officinalis.

J Chromatogr A. 2004; 1027: 121-126.

doi:10.1016/j.chroma.2003.11.046

[20] Carnat A, Carnat AP, Fraisse D, Ricoux L, Lamaison JL.

The aromatic and polyphenolic composition of Roman chamomile tea.

Fitoterapia. 2004; 75: 32-38.

doi:10.1016/j.fitote.2003.07.007

[21] Rosmarinblätter-Rosmarini folium.

In: Europäisches Arzneibuch (European Pharmacopoeia). 6th ed. Volume 3. Monographien K-Z.

Vienna: Verlag Österreich. 2008: 3865-3866. 
[22] XXII Allgemeine Bestimmungen Tropfentabelle In: Pharmacopoea Austriaca. Volume 1. Allgemeiner Teil, Amtliche Ausgabe 1990, 8. Nachtrag. Vienna: Verlag Österreich. 2001: 2.

[23] Bilia AR, Flamini G, Taglioli V, Morelli I, Vincieri FF.

GC-MS analysis of essential oil of some commercial Fennel teas.

Food Chem. 2002; 76: 307-310.

doi:10.1016/S0308-8146(01)00277-1

[24] Adams RP.

Identification of essential oil components by gas chromatographie/mass spectrometry. 4th ed. Carol Stream, Illinois: Allured Publishing Corporation, 2007.

[25] NIST 05.

Mass spectral library.

Gaithersburg: US National Institute of Standards and Technology, 2005.

[26] Oehme M.

Praktische Einführung in die GC/MS-Analytik mit Quadrupolen: Grundlagen und Anwendungen. Heidelberg: Hüthig Verlag, 1996: 138. 